\title{
Multistep, Microwave Assisted, Solvent Free Synthesis and Antibacterial Activity of 6-Substituted-2,3,4-trihydropyrimido[1,2-c]9,10,11,12- tetrahydrobenzo[b]thieno[3,2-e]pyrimidines
}

\author{
Mailavaram Raghu Prasad* and Deb Pran Kishore \\ Medicinal Chemistry Division, P.E.S. College of Pharmacy; Hanumanthnagar, Bangalore-560 050, Karnataka, India.
}

Received January 17, 2007; accepted February 19, 2007

A novel, efficient, microwave assisted route for the synthesis of 6-substituted-2,3,4-trihydropyrimido[1,2-c]9,10,11,12-tetrahydrobenzo[b]thieno[3,2-e]pyrimidines in good yields has been developed. The intermediates, 2substituted-4-[3-hydroxy(propyl-1-amino)]5,6,7,8-tetrahydrobenzo[b]thieno[2,3- $d]$ pyrimidines were obtained by irradiating 2-substituted-4-chloro-5,6,7,8-tetrahydrobenzo $[b]$ thieno $[2,3-d]$ pyrimidines with 1-amino-propanol under basic conditions in a microwave oven. 4-Chlorothieno[2,3-d]pyrimidines were synthesized by microwave irradiation of equimolar mixture of 4-hydroxythieno[2,3-d]pyrimidines and phosphorus oxychloride. The final compounds were screened for antibacterial activity by Kirby Bauer's method using amicacin as the standard against various gram positive and gram negative bacteria. All the compounds showed antibacterial activity comparable with the standard. method

Key words pyrimidothienopyrimidine; microwave assisted synthesis; green chemistry; solvent free synthesis; Kirby Bauer's

Antibacterial play an important role in the treatment of various bacterial infections. The spectacular success of antibiotics in the treatment of bacterial infection has prompted the expansion of their use from tetracycline's to fluroquinolines. ${ }^{1)}$ However, the emergence of resistant strains, even to fluroquinolines, has posed a real challenge. ${ }^{2)}$ Thus researchers are working towards finding new drugs by utilizing the concept of bioisosterism, to defeat resistant strains. ${ }^{3)}$

Literature survey revealed that several fused pyrimidines and pyridines like triazolo quinazolines and triazoloquinolines ${ }^{4)}$ have shown good antibacterial activity. Further condensed triazoles have been reported to possess large number of pharmacological activities like fungicidal, pesticidal etc. ${ }^{5-8)}$ Thienopyrimidines has been reported to exhibit antimicrobial activities too. ${ }^{9)}$ Thus it was taught of interest to fuse various heterocyclic moieties like imidazole, triazole ring system to the basic thienopyrimidine ring system and to test their efficacy for their antibacterial activity. Recently, triazolothienopyrimidines have been reported as antibacterial agents from our laboratories. ${ }^{10,11)}$ The encouraging antibacterial activity of these compounds gave us an impetus for isosteric replacement of triazole ring in triazolothienopyrimidines by pyrimidine ring. Thus synthesis of some novel pyrimidothienopyrimidines has been worked out.

Since 1986, with the introduction of controlled, precise microwave reactors, microwave-assisted organic synthesis has had a significant impact on synthetic organic chemistry. ${ }^{12)}$ Thus microwave assisted synthesis has gained popularity due to their enhanced selectivity, improved reaction rates, associated ease of manipulation and ecofriendliness.

Literature survey shows that microwaves are utilized for the synthesis of various heterocyclic compounds like quinolines ${ }^{13)}$ pyrazolopyrazoles,${ }^{14)}$ xanthines, ${ }^{15)}$ hydantoins, ${ }^{16)}$ benzoxazines, ${ }^{17)}$ quinazolines, ${ }^{18)}$ imidazolothienopyrimidines, ${ }^{10)}$ thiophenes, ${ }^{19)}$ thieno pyrimidines ${ }^{20)}$ etc. but no efforts were made to utilize microwaves for the synthesis of pyrimido$[1,2-c]$ thieno[3,2-e]pyrimidines. ${ }^{21,22)}$ These observations prompted us to attempt the synthesis of these compounds by microwave technique. Herewith we are reporting a novel microwave assisted synthesis and antibacterial activity of 6substituted-2,3,4-trihydropyrimido[1,2-c]9,10,11,12-tetrahydrobenzo[ $b]$ thieno[3,2-e]pyrimidines (Chart 1 ).

\section{Experimental}

Chemistry All reactions were carried out on microwave oven at the power of $960 \mathrm{~W}$ [CEM, Discover microwave labstation operating at $2450 \mathrm{MHz}$ under continuous internal temperature control]. Analytical TLC was performed on Silica Gel $\mathrm{F}_{254}$ plates (Merck) with visualization by UV or iodine vapors. Melting points were determined in open capillaries on a Thermonik melting point apparatus, Mumbai, India and are uncorrected. The IR spectra $\left(\mathrm{KBr}, v \mathrm{Max}, \mathrm{cm}^{-1}\right)$ were run on Perkin Elmer FTIR Spectrophotometer $(577$ model $) .{ }^{1} \mathrm{H}-\mathrm{NMR}\left(\delta \mathrm{ppm}, \mathrm{CDCl}_{3} / \mathrm{DMSO}-d_{6}\right)$ spectra were recorded using Bruker WM-400 spectrometer (Bruker, Flawil, Switzerland)
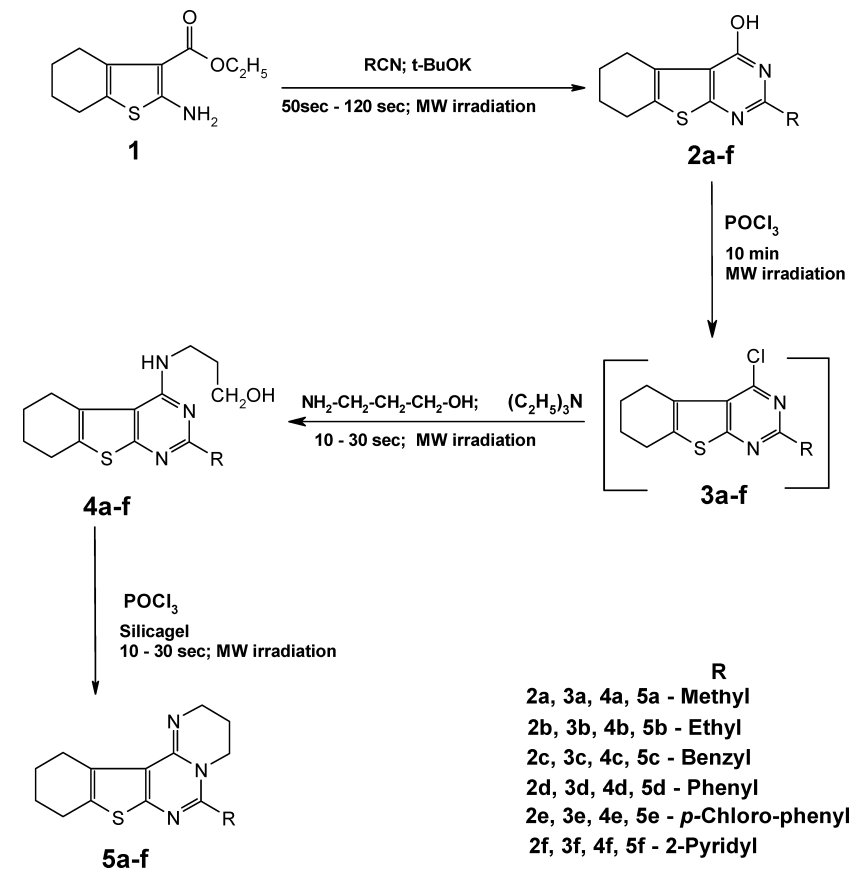

Chart 1. Microwave Assisted Synthesis of 6-Substituted-2,3,4-trihydropyrimido[1,2-c]9,10,11,12-tetrahydrobenzo[b]thieno[3,2-e]pyrimidines $(\mathbf{5 a}-\mathbf{f})$ 
with TMS as internal standard. MS spectra (EI-MS, $70 \mathrm{eV}$ ) were recorded on Autospec spectrometer. Elemental analyses were performed on Carlo Erba 1108 elemental analyzer (Heraeus, Hanau, Germany) and were within $\pm 0.4 \%$ of theoretical values. All the chemicals used were of analytical grade.

2-Substituted-5,6,7,8-tetrahydrobenzo $[b]$ thieno $[2,3-d]$ pyrimidin-4[3H]ones $(2 \mathbf{a}-\mathbf{f})$. General Procedure 2-Amino-3-carbethoxy-4,5,6,7-tetrahydrobenzo $[b]$ thiophene $1(8.3 \mathrm{mmol})$, potassium tert-butoxide $(0.089 \mathrm{~g}, 0.8$ $\mathrm{mmol})$ and various nitriles $(16 \mathrm{mmol})$ were taken in a $5 \mathrm{ml}$ microwave reaction vial equipped with a magnetic stir bar. The reaction mixture was irradiated in the microwave oven at $120^{\circ} \mathrm{C}$ for $45-150 \mathrm{~s}$. To the reaction mixture at room temperature, crushed ice was added and neutralized using dilute hydrochloric acid. The precipitate obtained was filtered, dried and recrystalized from ethylacetate to give the target compounds in $50-80 \%$ yields.

2-(p-Chlorobenzyl)-5,6,7,8-tetrahydrobenzo $[b]$ thieno $[2,3-d]$ pyrimidin4[3H]-one (2e) Reaction time: $150 \mathrm{~s}$. Yield 75\%; colorless needles; mp $286-288^{\circ} \mathrm{C}$; IR $(\mathrm{KBr}) \mathrm{cm}^{-1}: 1654(-\mathrm{C}=\mathrm{O}), 3417(-\mathrm{NH}), 2921\left(-\mathrm{CH}_{2}\right)$ $3008(\mathrm{Ar}-\mathrm{H}), 1046(-\mathrm{C}-\mathrm{N}) .{ }^{1} \mathrm{H}-\mathrm{NMR}\left(\mathrm{CDCl}_{3}\right) \delta: 1.75-1.95(4 \mathrm{H}, \mathrm{m})$, $2.65-2.95(4 \mathrm{H}, \mathrm{m}), 4.12(2 \mathrm{H}, \mathrm{s}), 8.34-9.15(4 \mathrm{H}, \mathrm{m}), 11.4(1 \mathrm{H}, \mathrm{br}-\mathrm{s})$. MS $m / z$ : $331.5\left(\mathrm{M}^{+}+1\right)$. Anal. Calcd for $\mathrm{C}_{17} \mathrm{H}_{15} \mathrm{~N}_{2} \mathrm{SOCl}$ : C, 61.72; H, 4.53; N 8.47. Found: C, $61.35 ; \mathrm{H}, 4.45 ; \mathrm{N}, 8.10$.

2-(2-Pyridyl)-5,6,7,8-tetrahydrobenzo $[b]$ thieno $[2,3-d]$ pyrimidin-4[3H]one (2f) Reaction time: $150 \mathrm{~s}$. Yield $72 \%$; colorless needles; mp 302 $304{ }^{\circ} \mathrm{C}$; IR (KBr) cm ${ }^{-1}$ : $3470(\mathrm{NH}), 3010(\mathrm{Ar}), 1670\left(\mathrm{C}=\mathrm{O} .{ }^{1} \mathrm{H}-\mathrm{NMR}\right.$ $\left(\mathrm{CDCl}_{3}\right) \delta: 1.68-1.89(4 \mathrm{H}, \mathrm{m}), 2.67-2.98(4 \mathrm{H}, \mathrm{m}), 8.21-9.10(4 \mathrm{H}, \mathrm{m})$ $11.2\left(1 \mathrm{H}\right.$, br-s, $\mathrm{D}_{2} \mathrm{O}$ exchangeable). MS $m / z: 284\left(\mathrm{M}^{+}+1\right)$. Anal. Calcd for $\mathrm{C}_{15} \mathrm{H}_{13} \mathrm{~N}_{3} \mathrm{SO}$ : C, 63.60; H, 4.59; N, 14.84. Found: C, 63.35; H, 4.85; N 15.10

2-Substituted-4-chloro-5,6,7,8-tetrahydrobenzo $[b]$ thieno[2,3- $d]$ pyrimidines 3a-f. General Procedure A mixture of compound $\mathbf{2 a}-\mathbf{f}$ (5 $\mathrm{mmol}$ ) and phosphorus oxychloride $(5 \mathrm{mmol})$ were taken in a $5 \mathrm{ml} \mathrm{mi}$ crowave reaction vial equipped with a magnetic stir bar. The reaction mixture was irradiated in the microwave at $120^{\circ} \mathrm{C}$ for $10 \mathrm{~min}$. The reaction mix ture was poured into crushed ice and neutralized with sodium bicarbonate solution. The solid thus obtained was collected and dried. The compound was taken to next step immediately without any purification.

2-Substituted-4-[(3-hydroxy)propyl]amino-5,6,7,8-tetrahydrobenzo[b]thieno[2,3-d]pyrimidines $4 \mathbf{a}-\mathbf{f}$. General Procedure A mixture of compound $\mathbf{3 a}-\mathbf{f}(5 \mathrm{mmol})$, 3-aminopropanol $(5 \mathrm{mmol})$ and triethylamine $(0.01 \mathrm{ml})$ were taken in a $5 \mathrm{ml}$ microwave reaction vial equipped with a magnetic stir bar. The reaction mixture was irradiated in the microwave oven at $120^{\circ} \mathrm{C}$ for 10 to $30 \mathrm{~s}$. The reaction mixture was poured into a beaker containing crushed ice and neutralized with dilute hydrochloric acid. The solid thus obtained was filtered, dried and crystallized from toluene to give the target compounds $\mathbf{4 a -} \mathbf{-}$ in good yields.

2-Methyl-4-[(3-hydroxy)propyl]amino-5,6,7,8-tetrahydrobenzo $[b]-$ thieno[2,3-d]pyrimidine (4a) Reaction time: $30 \mathrm{~s}$. Yield $91 \%$; colorless needles; mp 152-154 ${ }^{\circ} \mathrm{C}$; IR $(\mathrm{KBr}) \mathrm{cm}^{-1}$ : $3394(-\mathrm{OH}), 3243(-\mathrm{NH}), 2926$ $\left(-\mathrm{CH}_{2}\right), 3014\left(\mathrm{CH}_{3}\right), 1076(-\mathrm{C}-\mathrm{N}) .{ }^{1} \mathrm{H}-\mathrm{NMR}\left(\mathrm{CDCl}_{3}\right) \delta: 1.75-1.82(4 \mathrm{H}$, m), $1.94-2.01(2 \mathrm{H}$, pentet, $J=5.7 \mathrm{~Hz}),, 2.35(3 \mathrm{H}, \mathrm{S}), 2.69-2.71(2 \mathrm{H}, \mathrm{t}$, $J=5.9 \mathrm{~Hz}), 2.91-2.94(2 \mathrm{H}, \mathrm{t}, J=4.8 \mathrm{~Hz}), 3.49-3.56(2 \mathrm{H}, \mathrm{t}, J=5.8 \mathrm{~Hz})$, $3.83-3.86(2 \mathrm{H}, \mathrm{t}, J=5.7 \mathrm{~Hz}), 5.03(1 \mathrm{H}, \mathrm{s}), 5.52(1 \mathrm{H}, \mathrm{s})$. MS $m / z: 278$ $\left(\mathrm{M}^{+}+1\right)$. Anal. Calcd for $\mathrm{C}_{14} \mathrm{H}_{19} \mathrm{~N}_{3} \mathrm{SO}: \mathrm{C}, 60.34 ; \mathrm{H}, 6.55 ; \mathrm{N}, 14.96$. Found: C, 60.64; H, 6.85; N, 15.16 .

2-Ethyl-4-[(3-hydroxy)propyl]amino-5,6,7,8-tetrahydrobenzo $[b]-$ thieno[2,3-d]pyrimidine (4b) Reaction time: $30 \mathrm{~s}$. Yield $60 \%$; colorless needles; mp 144-146 ${ }^{\circ} \mathrm{C}$; IR ( $\left.\mathrm{KBr}\right) \mathrm{cm}^{-1}$ : $3394(-\mathrm{OH}), 3243(-\mathrm{NH}), 2926$ $\left(-\mathrm{CH}_{2}\right), 3014\left(\mathrm{CH}_{3}\right), 1076(-\mathrm{C}-\mathrm{N}) .{ }^{1} \mathrm{H}-\mathrm{NMR}\left(\mathrm{CDCl}_{3}\right) \delta: 1.38-1.43(3 \mathrm{H}, \mathrm{t}$, $J=7.5 \mathrm{~Hz}), 1.85-1.99(4 \mathrm{H}, \mathrm{m}), 2.19-2.29(2 \mathrm{H}$, pentet, $J=6.2 \mathrm{~Hz}), 2.75-$ $2.85(2 \mathrm{H}, \mathrm{t}, J=5.7 \mathrm{~Hz}), 2.93-2.95(2 \mathrm{H}, \mathrm{t}, J=5.6 \mathrm{~Hz}), 3.02-3.08(2 \mathrm{H}, \mathrm{q}$ $J=7.4 \mathrm{~Hz}), 3.67-3.71(2 \mathrm{H}, \mathrm{t}, J=6.1 \mathrm{~Hz}), 3.87-3.95(2 \mathrm{H}, \mathrm{t}, J=6.0 \mathrm{~Hz})$, $5.49(1 \mathrm{H}, \mathrm{s})$. MS m/z: $292\left(\mathrm{M}^{+}+1\right)$. Anal. Calcd for $\mathrm{C}_{15} \mathrm{H}_{21} \mathrm{~N}_{3} \mathrm{SO}: \mathrm{C}, 61.76$; H, 7.05; N, 14.10. Found: C, 61.85; H, 7.21; N, 14.43.

2-Benzyl-4-[(3-hydroxy)propyl] amino-5,6,7,8-tetrahydrobenzo $[b]$ thieno[2,3-d]pyrimidine (4c) Reaction time: $25 \mathrm{~s}$. Yield $92 \%$; colorless needles; mp 206-208 ${ }^{\circ}$; IR (KBr) cm ${ }^{-1}$ : $3400(-\mathrm{OH}), 3355(-\mathrm{NH}), 2924$ $\left(-\mathrm{CH}_{2}\right), 3016(\mathrm{Ar}-\mathrm{H}), 1048(-\mathrm{C}-\mathrm{N}), 1650(-\mathrm{C}=\mathrm{C}-) .{ }^{1} \mathrm{H}-\mathrm{NMR}\left(\mathrm{CDCl}_{3}\right) \delta$ : $1.88-1.98(4 \mathrm{H}, \mathrm{m}), 2.23-2.30(2 \mathrm{H}$, pentet, $J=6.2 \mathrm{~Hz}), 2.74-2.82(2 \mathrm{H}, \mathrm{t}$ $J=5.7 \mathrm{~Hz}), 2.91-2.96(2 \mathrm{H}, \mathrm{t}, J=5.7 \mathrm{~Hz}), 3.65-3.74(2 \mathrm{H}, \mathrm{t}, J=6.2 \mathrm{~Hz})$, $3.88-3.92(2 \mathrm{H}, \mathrm{t}, J=6.2 \mathrm{~Hz}), 4.11(2 \mathrm{H}, \mathrm{s}), 5.47(1 \mathrm{H}, \mathrm{s}), 7.41-7.50(5 \mathrm{H}$ m). MS $m / z$ : $354\left(\mathrm{M}^{+}+1\right)$. Anal. Calcd for $\mathrm{C}_{20} \mathrm{H}_{23} \mathrm{~N}_{3} \mathrm{SO}: \mathrm{C}, 67.66 ; \mathrm{H}, 6.25$; N, 11.65. Found: C, 67.98; H, 6.51; N, 11.89 .

2-Phenyl-4-[(3-hydroxy)propyl]amino-5,6,7,8-tetrahydrobenzo $[b]-$ thieno[2,3-d]pyrimidine (4d) Reaction time: $20 \mathrm{~s}$. Yield $90 \%$; colorless needles; mp $154-156^{\circ} \mathrm{C}$; IR (KBr) $\mathrm{cm}^{-1}$ : $3393(-\mathrm{OH}), 3200(-\mathrm{NH}), 2923$ $\left(-\mathrm{CH}_{2}\right), 1655(-\mathrm{C}=\mathrm{C}-), 1066(-\mathrm{C}-\mathrm{N}) .{ }^{1} \mathrm{H}-\mathrm{NMR}\left(\mathrm{CDCl}_{3}\right) \delta: 1.81-1.95$ $(4 \mathrm{H}, \mathrm{m}), 2.23-2.30(2 \mathrm{H}$, pentet, $J=6.1 \mathrm{~Hz}), 2.72-2.84(2 \mathrm{H}, \mathrm{t}, J=5.7 \mathrm{~Hz})$, $2.92-2.94(2 \mathrm{H}, \mathrm{t}, J=5.7 \mathrm{~Hz}), 3.64-3.72(2 \mathrm{H}, \mathrm{t}, J=6.2 \mathrm{~Hz}), 3.88-3.92$ $(2 \mathrm{H}, \mathrm{t}, J=6.2 \mathrm{~Hz}), 5.47(1 \mathrm{H}, \mathrm{s}), 7.41-7.50(3 \mathrm{H}, \mathrm{m}), 8.44-8.52(2 \mathrm{H}, \mathrm{d}$ $J=6.3 \mathrm{~Hz})$. MS $m / z: 340\left(\mathrm{M}^{+}+1\right)$. Anal. Calcd for $\mathrm{C}_{19} \mathrm{H}_{21} \mathrm{~N}_{3} \mathrm{SO}: \mathrm{C}, 66.95$; H, 5.85; N, 12.05. Found: C, 67.25; H, 6.19; N, 12.38.

2-(p-Chlorobenzyl)]-4-[(3-hydroxy)propyl]amino-5,6,7,8-tetrahydrobenzo $[\boldsymbol{b}]$ thieno $[2,3-\boldsymbol{d}]$ pyrimidine (4e) Reaction time: $15 \mathrm{~s}$. Yield $88 \%$ colorless needles; mp $230-232^{\circ} \mathrm{C}$; IR $(\mathrm{KBr}) \mathrm{cm}^{-1}$ : $3400(-\mathrm{OH}), 3355$ $(-\mathrm{NH}), 2924\left(-\mathrm{CH}_{2}\right), 3016(\mathrm{Ar}-\mathrm{H}), 1048(-\mathrm{C}-\mathrm{N}), 1650(-\mathrm{C}=\mathrm{C}-) .{ }^{1} \mathrm{H}-\mathrm{NMR}$ $\left(\mathrm{CDCl}_{3}\right) \delta: 1.85-2.15(4 \mathrm{H}, \mathrm{m}), 2.25-2.30(2 \mathrm{H}$, pentet, $J=6.3 \mathrm{~Hz}), 2.85-$ $2.88(2 \mathrm{H}, \mathrm{t}, J=5.9 \mathrm{~Hz}), 2.95-2.98(2 \mathrm{H}, \mathrm{t}, J=5.9 \mathrm{~Hz}), 3.65-3.70(2 \mathrm{H}, \mathrm{t}$ $J=6.2 \mathrm{~Hz}), 3.90-3.93(2 \mathrm{H}, \mathrm{t}, J=6.2 \mathrm{~Hz}), 4.14(2 \mathrm{H}, \mathrm{s}), 5.62(1 \mathrm{H}, \mathrm{s}), 8.30$ $8.32(2 \mathrm{H}, \mathrm{d}, J=3.6 \mathrm{~Hz}), 8.55-8.75(2 \mathrm{H}, \mathrm{d}, J=3.7 \mathrm{~Hz})$. MS $m / z: 388.5$ $\left(\mathrm{M}^{+}+1\right)$. Anal. Calcd for $\mathrm{C}_{20} \mathrm{H}_{22} \mathrm{~N}_{3} \mathrm{SOCl}$ : C, 61.65; H, 5.35; N, 10.57 . Found: C, 61.93; H, 5.67; N, 10.83

2-(2-Pyridyl)-4-[(3-hydroxy)propyl] amino-5,6,7,8-tetrahydrobenzo $[b]$ thieno[2,3-d]pyrimidine (4f) Reaction time: $10 \mathrm{~s}$. Yield $87 \%$; colorless needles; mp 205-206 ${ }^{\circ} \mathrm{C}$; IR (KBr) cm ${ }^{-1}$ : $3366(-\mathrm{OH}), 3126(-\mathrm{NH}), 2946$ $\left(-\mathrm{CH}_{2}\right), 3014(\mathrm{Ar}-\mathrm{H}), 1071(-\mathrm{C}-\mathrm{N}) .{ }^{1} \mathrm{H}-\mathrm{NMR}\left(\mathrm{CDCl}_{3}\right) \delta: 1.83-2.17(4 \mathrm{H}$ m), $2.23-2.29(2 \mathrm{H}$, pentet, $J=6.2 \mathrm{~Hz}), 2.84-2.86(2 \mathrm{H}, \mathrm{t}, J=5.8 \mathrm{~Hz})$, $2.93-2.95(2 \mathrm{H}, \mathrm{t}, J=5.8 \mathrm{~Hz}), 3.69-3.72(2 \mathrm{H}, \mathrm{t}, J=6.1 \mathrm{~Hz}), 3.88-3.93$ $(2 \mathrm{H}, \mathrm{t}, J=6.2 \mathrm{~Hz}), 5.56(1 \mathrm{H}, \mathrm{s}), 8.28-8.29(2 \mathrm{H}, \mathrm{d}, J=3.4 \mathrm{~Hz}), 8.56-8.71$ $(2 \mathrm{H}, \mathrm{d}, J=3.5 \mathrm{~Hz})$. MS m/z: $341\left(\mathrm{M}^{+}+1\right)$. Anal. Calcd for $\mathrm{C}_{18} \mathrm{H}_{20} \mathrm{~N}_{4} \mathrm{SO}: \mathrm{C}$ 63.15; H, 5.65; N, 16.17. Found: C, 63.52; H, 5.88; N, 16.47 .

6-Substituted-2,3,4-trihydropyrimido $[1,2-c] 9,10,11,12$-tetrahydrobenzo[b]thieno[3,2-e]pyrimidines 5a-f. General Procedure Compound 4af $(5 \mathrm{mmol})$ coated on to silica gel \# 60/120 was taken into a $5 \mathrm{ml}$ microwave reaction vial equipped with a magnetic stir bar. To the coated silica gel, phosphorus oxychloride $(5 \mathrm{mmol})$ was added. The reaction vessel was capped and irradiated in the microwave oven (CEM, Discover) at $120^{\circ} \mathrm{C}$ for 10 to $30 \mathrm{~s}$. The reaction mixture was poured into crushed ice and neutralized with sodium bicarbonate. The compound thus obtained was extracted with ethylacetate, dried and distilled of the excess of solvent to get the target compounds $\mathbf{5 a}-\mathbf{f}$ in good yields. The compound was purified by recrystallization from benzene.

6-Methyl-2,3,4-trihydropyrimido[1,2-c $] 9,10,11,12$-tetrahydrobenzo $[b]$ thieno[3,2-e]pyrimidine (5a) Reaction time: $15 \mathrm{~s}$. Yield $85 \%$; colorless needles; mp 174-176 ${ }^{\circ} \mathrm{C}$; IR $(\mathrm{KBr}) \mathrm{cm}^{-1}: 2926\left(-\mathrm{CH}_{2}\right), 3014\left(-\mathrm{CH}_{3}\right), 1620$ $(-\mathrm{C}=\mathrm{C}-) .{ }^{1} \mathrm{H}-\mathrm{NMR}\left(\mathrm{CDCl}_{3}\right) \delta: 1.64-1.66(2 \mathrm{H}$, pentet, $J=4.9 \mathrm{~Hz}), 1.71-$ $1.73(2 \mathrm{H}$, pentet, $J=4.9 \mathrm{~Hz}), 1.79-1.83(2 \mathrm{H}$, pentet, $J=5.9 \mathrm{~Hz}), 2.28(3 \mathrm{H}$, s), $2.62-2.63(2 \mathrm{H}, \mathrm{t}, J=4.8 \mathrm{~Hz}), 2.79-2.82(2 \mathrm{H}, \mathrm{t}, J=4.8 \mathrm{~Hz}), 3.34-3.36$ $(2 \mathrm{H}, \mathrm{t}, J=5.9 \mathrm{~Hz}), 3.82-3.85(2 \mathrm{H}, \mathrm{t}, J=5.8 \mathrm{~Hz})$. MS $m / z: 260\left(\mathrm{M}^{+}+1\right)$. Anal. Calcd for $\mathrm{C}_{14} \mathrm{H}_{17} \mathrm{~N}_{3} \mathrm{~S}$ : C, 64.55; H, 6.15; N, 15.98. Found: C, 64.86; H, $6.56 ; \mathrm{N}, 16.21$.

6-Ethyl-2,3,4-trihydropyrimido $[1,2-c] 9,10,11,12$-tetrahydrobenzo $[b]-$ thieno[3,2-e]pyrimidine (5b) Reaction time: $20 \mathrm{~s}$. Yield 55\%; colorless needles; mp $176-178^{\circ} \mathrm{C}$; IR $(\mathrm{KBr}) \mathrm{cm}^{-1}$ : 3014 (alkyl), $1076(\mathrm{C}-\mathrm{N}) .{ }^{1} \mathrm{H}-$ $\operatorname{NMR}\left(\mathrm{CDCl}_{3}\right) \delta: 1.38-1.43(3 \mathrm{H}, \mathrm{t}, J=7.5 \mathrm{~Hz}), 1.85-1.99(4 \mathrm{H}, \mathrm{m}), 2.18$ $2.28(2 \mathrm{H}$, pentet, $J=6.2 \mathrm{~Hz}), 2.75-2.85(2 \mathrm{H}, \mathrm{t}, J=5.7 \mathrm{~Hz}), 2.92-2.98(2 \mathrm{H}$, $\mathrm{t}, J=5.6 \mathrm{~Hz}), 3.02-3.08(2 \mathrm{H}, \mathrm{q}, J=7.5 \mathrm{~Hz}), 3.63-3.73(2 \mathrm{H}, \mathrm{t}, J=6.0 \mathrm{~Hz})$, 3.87-3.97 $(2 \mathrm{H}, \mathrm{t}, J=6.0 \mathrm{~Hz})$. MS $m / z: 274\left(\mathrm{M}^{+}+1\right)$. Anal. Calcd for $\mathrm{C}_{15} \mathrm{H}_{19} \mathrm{~N}_{3} \mathrm{~S}$ : C, 65.76; H, 6.65; N, 15.20. Found: C, 65.93; H, 6.95; N, 15.38 .

6-Benzyl-2,3,4-trihydropyrimido $[1,2-c] 9,10,11,12$-tetrahydrobenzo $[b]$ thieno[3,2-e]pyrimidine (5c) Reaction time: $30 \mathrm{~s}$. Yield 70\%; colorless needles; mp $120-122^{\circ} \mathrm{C}$; IR $(\mathrm{KBr}) \mathrm{cm}^{-1}: 2933\left(-\mathrm{CH}_{2}\right), 3025(\mathrm{Ar}-\mathrm{H}), 1621$ $(\mathrm{C}=\mathrm{C}), 1071(\mathrm{C}-\mathrm{N}) .{ }^{1} \mathrm{H}-\mathrm{NMR}\left(\mathrm{CDCl}_{3}\right) \delta: 2.16-2.20(4 \mathrm{H}, \mathrm{m}), 2.49-2.53$ $(2 \mathrm{H}$, pentet, $J=6.4 \mathrm{~Hz}), 2.79(2 \mathrm{H}, \mathrm{t}, J=5.8 \mathrm{~Hz}), 2.98(2 \mathrm{H}, \mathrm{t}, J=5.9 \mathrm{~Hz})$, $3.73-3.77(4 \mathrm{H}, \mathrm{m}), 4.10(2 \mathrm{H}, \mathrm{s}), 7.45-8.25(5 \mathrm{H}, \mathrm{m}) . \mathrm{MS} m / z: 336$ $\left(\mathrm{M}^{+}+1\right)$. Anal. Calcd for $\mathrm{C}_{20} \mathrm{H}_{21} \mathrm{~N}_{3} \mathrm{~S}: \mathrm{C}, 71.35 ; \mathrm{H}, 6.21 ; \mathrm{N}, 13.31$. Found: $\mathrm{C}$, 71.64; H, 6.26; N, 12.53 .

6-Phenyl-2,3,4-trihydropyrimido $[1,2-c] 9,10,11,12$-tetrahydrobenzo $[b]$ thieno[3,2-e]pyrimidine (5d) Reaction time: $20 \mathrm{~s}$. Yield $80 \%$; colorless needles; mp $168-170{ }^{\circ} \mathrm{C}$; IR $(\mathrm{KBr}) \mathrm{cm}^{-1}$ : $2929\left(-\mathrm{CH}_{2}\right), 3014$ (Ar-H), 1655 $(\mathrm{C}=\mathrm{C}), 1048(\mathrm{C}-\mathrm{N}) .{ }^{1} \mathrm{H}-\mathrm{NMR}\left(\mathrm{CDCl}_{3}\right) \delta: 2.13-2.19(4 \mathrm{H}, \mathrm{m}), 2.48-2.51$ $(2 \mathrm{H}$, pentet, $J=6.4 \mathrm{~Hz}), 2.77(2 \mathrm{H}, \mathrm{t}, J=5.7 \mathrm{~Hz}), 2.96(2 \mathrm{H}, \mathrm{t}, J=5.7 \mathrm{~Hz})$, $3.73-3.77(4 \mathrm{H}, \mathrm{m}), 7.45-7.55(3 \mathrm{H}, \mathrm{m}), 8.37-8.39(2 \mathrm{H}, \mathrm{d}, J=4.9 \mathrm{~Hz}) . \mathrm{MS}$ $m / z: 322\left(\mathrm{M}^{+}+1\right)$. Anal. Calcd for $\mathrm{C}_{19} \mathrm{H}_{19} \mathrm{~N}_{3} \mathrm{~S}: \mathrm{C}, 71.35 ; \mathrm{H}, 6.21 ; \mathrm{N}, 13.31$. Found: C, 71.02; H, 5.91; N, 13.08

6-(p-Chlorobenzyl)-2,3,4-trihydropyrimido[1,2-c]9,10,11,12-tetrahydrobenzo $[\boldsymbol{b}]$ thieno[3,2-e]pyrimidine $(\mathbf{5 e})$ Reaction time: $10 \mathrm{~s}$. Yield 68 $\%$; colorless needles; mp 90-92 ${ }^{\circ} \mathrm{C}$; IR $(\mathrm{KBr}) \mathrm{cm}^{-1}$ : $2933\left(-\mathrm{CH}_{2}\right), 3025$ (Ar-H), $1621(\mathrm{C}=\mathrm{C}), 1071(\mathrm{C}-\mathrm{N}) .{ }^{1} \mathrm{H}-\mathrm{NMR}\left(\mathrm{CDCl}_{3}\right) \delta: 1.94-2.28(4 \mathrm{H}$, 
m), $2.33-2.38(2 \mathrm{H}$, pentet, $J=6.4 \mathrm{~Hz}), 2.81-2.84(2 \mathrm{H}, \mathrm{t}, J=5.9 \mathrm{~Hz})$, $2.99-3.03(2 \mathrm{H}, \mathrm{t}, J=5.8 \mathrm{~Hz}), 3.76-3.80(4 \mathrm{H}, \mathrm{m}), 4.15(2 \mathrm{H}, \mathrm{s}), 8.25-8.28$ $(2 \mathrm{H}, \mathrm{d}, J=4.0 \mathrm{~Hz}), 8.72-8.74(2 \mathrm{H}, \mathrm{d}, J=3.9 \mathrm{~Hz}) . \mathrm{MS} m / z: 370.5\left(\mathrm{M}^{+}+1\right)$. Anal. Calcd for $\mathrm{C}_{20} \mathrm{H}_{20} \mathrm{~N}_{3} \mathrm{SCl}$ : C, 64.75; H, 5.25; N, 11.20. Found: C, 64.95; $\mathrm{H}, 5.41 ; \mathrm{N}, 11.36$.

6-(2-Pyridyl)-2,3,4-trihydropyrimido[1,2-c]9,10,11,12-tetrahydrobenzo[b]thieno[3,2-e]pyrimidine (5f) Reaction time: $25 \mathrm{~s}$. Yield 78\%; colorless needles; mp $146-148^{\circ} \mathrm{C}$; IR $(\mathrm{KBr}) \mathrm{cm}^{-1}$ : $2943\left(-\mathrm{CH}_{2}\right), 2997(\mathrm{Ar}-\mathrm{H}), 1060$ $(-\mathrm{C}-\mathrm{N}) .{ }^{1} \mathrm{H}-\mathrm{NMR}\left(\mathrm{CDCl}_{3}\right) \delta: 1.84-2.18(4 \mathrm{H}, \mathrm{m}), 2.13-2.18(2 \mathrm{H}$, pentet, $J=6.3 \mathrm{~Hz}), 2.79-2.83(2 \mathrm{H}, \mathrm{t}, J=5.8 \mathrm{~Hz}), 2.97-3.01(2 \mathrm{H}, \mathrm{t}, J=5.8 \mathrm{~Hz})$, $3.74-3.78(4 \mathrm{H}, \mathrm{m}), 8.23-8.24(2 \mathrm{H}, \mathrm{d}, J=3.8 \mathrm{~Hz}), 8.67-8.68(2 \mathrm{H}, \mathrm{d}$, $J=3.8 \mathrm{~Hz})$. MS $m / z: 323\left(\mathrm{M}^{+}+1\right)$. Anal. Calcd for $\mathrm{C}_{18} \mathrm{H}_{18} \mathrm{~N}_{4} \mathrm{~S}: \mathrm{C}, 66.85 ; \mathrm{H}$, 5.25; N, 17.10. Found: C, 67.08; H, 5.59; N, 17.39.

Antibacterial Activity-Kirby Bauer's Method Peptone water was prepared and autoclaved. Four broth cultures were prepared using peptone water containing one type of organism each from stock cultures. A sterile cotton swab was dipped into one of the broth cultures and used to inoculate a Mueller Hinton agar plate. Inoculation of the plate in this way ensured a lawn of bacterial growth after incubation. Repeated this inoculation procedure for four plates from four different broth cultures and the plates were labeled. After inoculation the plates were allowed to dry for $15 \mathrm{~min}$ before proceeding to the next step. $70 \%$ ethanol was poured into a $250 \mathrm{ml}$ beaker. The forceps was dipped into the alcohol and then passed the forceps over the Bunsen burner flame to sterilize it. The standard antibiotic disk (amikacin) was picked up and placed it in the centre of the plate. The whatman filter paper disc impregnated with newly synthesized drugs $(0.005 \mathrm{ml}, 50 \mu \mathrm{g})$ was picked and placed it in the corners. This procedure was repeated for the four plates for four newly synthesized drugs and incubated for $18 \mathrm{~h}$ at $35^{\circ} \mathrm{C}$. The plates were examined for zones of inhibition. They were measured with millimeter ruler across the disk. The diameter of the zone to the nearest whole millimeter was recorded. The disk impregnated with solvent (DMSO) and evaporated to dryness was used as negative control.

\section{Results and Discussion}

2-Amino-3-carbethoxy-4,5,6,7-tetrahydrobenzo[b]thiophene (1) was synthesized by following the reported procedures using microwave oven. ${ }^{19)}$ 2-Substituted-4-hydroxy5,6,7,8-tetrahydrobenzo[ $b]$ thieno[2,3- $d]$ pyrimidines $\quad(\mathbf{2 a}-\mathbf{f})$ were prepared by irradiating compound $\mathbf{1}$ with various aryl/alkyl nitriles in presence of catalytic amount of potassium-tert-butoxide at $120^{\circ} \mathrm{C}$ for 45 to $150 \mathrm{~s}$ under atmospheric pressure. ${ }^{11)}$ The melting points of the products obtained by microwave irradiation method were similar to that of reported one. The disappearance of two peaks of primary amino group and appearance of secondary amino peak in the range of 3200 to $3400 \mathrm{~cm}^{-1}$ and shift of carbonyl peak from $1724 \mathrm{~cm}^{-1}$ to $1650-1680 \mathrm{~cm}^{-1}$ indicated the cyclization of ortho amino ester of thiophene. The ${ }^{1} \mathrm{H}-\mathrm{NMR}$ spectra showed $\mathrm{D}_{2} \mathrm{O}$ exchangeable secondary amino signals as broad singlet at around $\delta 11.9 \mathrm{ppm}$ and mass spectra exhibited molecular ion peaks corresponding to the molecular weight of the compounds. The intermediate, 2-substituted-4-chloro-5,6,7,8tetrahydrobenzo $[b]$ thieno $[2,3-d]$ pyrimidines $\quad(\mathbf{3 a}-\mathbf{f}) \quad$ were prepared by irradiating the equimolar mixture of $\mathbf{2 a}-\mathbf{f}$ and phosphorus oxychloride for $10 \mathrm{~min}$ at $120^{\circ} \mathrm{C}$. The reaction mixture was worked up by adding onto crushed ice and neutralizing with sodium bicarbonate. The solid obtained was filtered, dried and used for next step immediately without any purification.

Equimolar mixtures of 2-substituted-4-chloro-5,6,7,8tetrahydrobenzo[ $b]$ thieno[2,3- $d]$ pyrimidines $\quad 3 \mathbf{a}-\mathbf{f}$, aminopropanol and triethylamine were irradiated in a microwave oven at $120^{\circ} \mathrm{C}$ for 10 to $30 \mathrm{~s}$ to give 2-substituted-4-[3-hydroxy(propyl-1-amino)]5,6,7,8-tetrahydrobenzo[b]thieno$[2,3-d]$ pyrimidines $(\mathbf{4 a}-\mathbf{f})$ in good yields $(80-90 \%)$. The IR spectrum of compounds $4 \mathbf{a}-\mathbf{f}$ showed a broad peak at $3400-3500 \mathrm{~cm}^{-1}$ for hydroxyl group and a peak at 3200 $3400 \mathrm{~cm}^{-1}$ for secondary amino group, which indicated the formation of the expected products. In the NMR spectrum, an exchangeable $\left(\mathrm{D}_{2} \mathrm{O}\right)$ amino signal at $\delta 6.0 \mathrm{ppm}$ and two triplets and one pentet for methylene protons of aminopropanol in the range of 3.7-3.6, 3.8-3.9, 2.3-2.2 ppm respectively confirmed the product formation. Further the mass spectrum exhibited a prominent molecular ion $\left(\mathrm{M}^{+}+1\right)$ and the isotopic peak $\left(\mathrm{M}^{+}+2\right)$ disappeared which confirmed the loss of chlorine in all the derivatives.

Compounds $4 \mathbf{a}-\mathbf{f}$ coated onto silica gel, underwent cyclization, in presence of equimolar quantities of phosphorus oxychloride under microwave irradiation, for 10 to $30 \mathrm{~s}$ at $120^{\circ} \mathrm{C}$ under normal atmospheric pressure to result in the target compounds $(\mathbf{5 a}-\mathbf{f})$ in good yields $(80-90 \%)$. The disappearance of peaks corresponding to amino and hydroxyl groups in the IR spectra and signals at $\delta 6.0 \mathrm{ppm}$ and $4.8 \mathrm{ppm}$ corresponding to $\mathrm{NH}$ and $\mathrm{OH}$, respectively, in their NMR spectra inferred product formation. The mass spectra of $\mathbf{5 a}-\mathbf{f}$, revealed the molecular ion $\left(\mathrm{M}^{+}+1\right)$ appearing as base peak $(100 \%)$. Based on the spectral data, the compounds, $\mathbf{5 a}-\mathbf{f}$, were characterized as 6-substituted-2,3,4-trihydropyrimido $[1,2-c] 9,10,11,12$-tetrahydrobenzo $[b]$ thieno$[3,2-e]$ pyrimidines. The elemental analyses of the newly synthesized compounds showed that the purity of the compounds were within $\pm 0.4 \%$ limits.

Thus the usage of present controlled, precise microwave reactor [CEM, Discover] has not only resulted in simple reaction conditions and easy work-up procedures but also improved yields over conventional methods. ${ }^{21,22)}$

The synthesized compounds were evaluated for antimicrobial activity against various gram-positive and gram-negative bacteria like K. pneumoniae, $P$. aeruginosa, B. subtilis and $S$. citrus using Kirby Bauer's Method. ${ }^{23)}$ The negative control did not show any zone of inhibition in all the bacterial strains used for the study. B. subtilis was found to be the most susceptible and $K$. pneumoniae was the most resistant organism.

Table 1. Antibacterial Activity of 6-Substituted-2,3,4-trihydropyrimido[1,2-c]9,10,11,12-tetrahydrobenzo[b]thieno[3,2-e]pyrimidines $(\mathbf{5 a}-\mathbf{f})$

\begin{tabular}{|c|c|c|c|c|c|}
\hline \multirow{2}{*}{ Compd. } & \multirow{2}{*}{ Concn. } & \multicolumn{4}{|c|}{ Zones of inhibition (in $\mathrm{mm}) \pm$ S.E.M. } \\
\hline & & K. pneumoniae & P. aeruginosa & B. subtilis & S. citrus \\
\hline $5 \mathbf{a}$ & $50 \mu \mathrm{g}$ & $10.0 \pm 0.42$ & $7.0 \pm 0.12$ & $12.0 \pm 0.14$ & $6.0 \pm 0.39$ \\
\hline $5 \mathbf{b}$ & $50 \mu \mathrm{g}$ & $11.0 \pm 0.36$ & $7.0 \pm 0.24$ & $11.0 \pm 0.30$ & $9.0 \pm 0.28$ \\
\hline $5 c$ & $50 \mu \mathrm{g}$ & $12.0 \pm 0.36$ & $7.0 \pm 0.31$ & $11.0 \pm 0.28$ & $7.0 \pm 0.62$ \\
\hline $5 d$ & $50 \mu \mathrm{g}$ & $11.0 \pm 0.28$ & $7.0 \pm 0.34$ & $10.0 \pm 0.13$ & $9.0 \pm 0.45$ \\
\hline $5 e$ & $50 \mu \mathrm{g}$ & $11.0 \pm 0.14$ & $7.0 \pm 0.24$ & $11.0 \pm 0.31$ & $7.0 \pm 0.54$ \\
\hline $5 f$ & $50 \mu \mathrm{g}$ & $11.0 \pm 0.18$ & $9.0 \pm 0.44$ & $11.0 \pm 0.31$ & $7.0 \pm 0.84$ \\
\hline Amikacin & $50 \mu \mathrm{g}$ & $20.0 \pm 0.35$ & $11.0 \pm 0.28$ & $12.0 \pm 0.25$ & $10.0 \pm 0.20$ \\
\hline
\end{tabular}


$P$. aeruginosa and $S$. citrus, showed intermediate activity.

All the compounds of the series $(\mathbf{5 a}-\mathbf{f})$ were found to be equipotent against with that of the standard against $B$. subtilis which indicates the susceptibility of the organism. Compounds $5 \mathbf{b}(9.0 \pm 0.45)$ and $\mathbf{5 d}(9.0 \pm 0.28)$ showed efficacy similar to that of amikacin $(10.0 \pm 0.20)$ against $S$. citrus. Compound 5 a showed least activity in the series.

The activity of the compounds $(\mathbf{5 a}-\mathbf{f})$ [zone of inhibition $10-12 \mathrm{~mm}$ ] against $K$. pneumoniae were not comparable with that of amikacin $(20 \pm 0.35)$ indicating the resistance of the organism. Compounds of the series were moderately active against $P$. aeruginosa. Compound 5 f $(9.0 \pm 0.44)$ was the most active of the series.

As all the compounds showed antibacterial activity against the bacteria tested, it indicates that this basic moiety can be a potential scaffold for antibacterial drugs. However B. subtilis was the only susceptible organism and other organisms were found to be bit resistant. Thus further lead optimization is required to get wide spectrum of activity.

Acknowledgements One of the authors (Raghu Prasad M.) is thankful to All India Council for Technical Education (AICTE) for granting Career Award for Young Teachers (CAYT). The necessary facilities extended by Management and Principal, P.E.S. College of Pharmacy, Hanumanthnagar, Bangalore-560 050 to carry out this work is gratefully acknowledged. We profusely thank Prof. Dr. Christa Mueller, Universitat Bonn, Pharmazeutisches Institut, Pharmazeutische Chemie, Poppelsdorf Kreuzbergweg 26 D53115 Bonn, Germany for providing facilities to carry out microwave assisted synthesis and Prof. Dr. Akkinepally Raghuram Rao, UCPSC, Panjab University, Chandigarh for moral support. We thank IISC, Bangalore for Spectral Data.

\section{References and Notes}

1) John M. B., "Wilson and Gisvold's, Text Book of Organic Medicinal and Pharmaceutical Chemistry," XI ed., Chap. 10, ed. by John H. B1., John M. Be., Jr., Lippincott Williams and Wilkins, A Wolters Kluwer Company, Philadelphia, 2004, pp. 299-300.

2) Keith S. K., Sara C., Anthony H., George M. E., Yehuda C., Antimicrob. Agents Chemother., 45, 2628-2630 (2001).

3) Wistreich G. A., Lechtman M. D., "Mechanism of drug resistance In
Microbiology," V ed., Macmallan Publishing Company, New York, 1988, pp. 353-354.

4) Savini L., Chiasserini L., Pellerano C., Filippelli W., Falcone G., Farmaco, 56, 939-945 (2001).

5) Leisner S., Guetschow M., Droessler K., Wagner G., Kluge S., Dieter L., Appl. 340044, Ger. Patent 293824 (1992) [Chem Abstr., 117, 48589k (1992)].

6) Edie R., Gerald H., Ronald E., Krumkains E., Viktors Appl. EP 452002 Eur. Patent 452002 (1992) [Chem. Abstr., 116, 21069h (1992)].

7) Obata I., Tokio F., Katsutoshi N., Isamu S., Shoji Appl. EP 356,158 Eur. Patent 356158, 1992 [Chem. Abstr., 113, 59210y (1990)].

8) Yamamoto S., Yokoi S., Matsumoto K., Honda T., Kobayashi T., Appl. EP 103,114. Eur. Patent 103114 (1984) [Chem. Abstr., 101, 23494g (1984)].

9) Chambhare R. V., Bobade A. S., Khadse B. G., Indian J. Heterocycl. Chem., 12, 67-70 (2002).

10) Raghu Prasad M., Rao A. R. R., Shanthan Rao P., Rajan K. S., Synthesis, 14, 2119-2123 (2001).

11) Raghu Prasad M., Prashanth J., Shilpa K., Pran Kishore D., Chem. Pharm. Bull., 55, 557-560 (2007).

12) Gedye R., Smith F., Westaway K., Ali H., Baldisera L., Tetrahedron Lett., 27, 279-282 (1986).

13) Annalisa M., Marisa M., Umberto P., Nickolas R., Stefania G., Tetrahedron Lett., 45, 6623-6627 (2004).

14) Satya P., Mukta G., Rajive G., Andre C., Tetrahedron Lett., 42, 3827 3829 (2001).

15) Leu Z. Y., John Z., Tetrahedron Lett., 47, 775-778 (2006).

16) Giulio G. M., Jacques H. P., Johan W., Bernadette N., Wolfgang P., Gerhard K. E. S., Didier M. L., Tetrahedron, 59, 1301-1307 (2003).

17) Wei-Min D., Xuan W., Chen Ma., Tetrahedron, 61, 6879-6885 (2005).

18) Julio A. S., Vazquez Tato M. P., Martinez M. M., Tetrahedron Lett., 41, 2215-2217 (2000).

19) Wei H., Jian L., Jing T., Hong L., Jianhau S., Hualiang J., Syn. Commun., 35, 1351-1357 (2005).

20) Yoon D. S., Han Y., Stark T. M., Haber J. C., Gregg B. T., Stankovich S. B., Org. Lett., 6, 4775-4778 (2004).

21) Leistner S., Geutschow M., Viewag H., Wahner G., Strob H., Grupe R., Pharmazie, 43, 756-760 (1988).

22) Leistner S., Geutschow M., Viewag H., Wahner G., Strob H., Grupe R., Lohmann D., Laban G., Ger. Patent 258233 (1988).

23) Bauer A. W., Kirby W. M. M., Sherris J. C., Tuck M., Am. J. Clin. Pathol., 45, 493-496 (1966). 